\title{
A New Spectral Method on Triangles
}

\author{
Youyun Li, Li-Lian Wang, Huiyuan Li, and Heping Ma
}

\begin{abstract}
We propose in this note a spectral method on triangles based on a new rectangle-to-triangle mapping, which leads to more reasonable grid distributions and efficient implementations than the usual approaches based on the collapsed transform. We present the detailed implementation for spectral approximations on a triangle and discuss the extension to spectral-element methods and three dimensions.
\end{abstract}

\section{Introduction}

Spectral element methods, which are capable of extending the standard spectral methods to complex geometries, have become an important tool for simulations of fluid dynamics, atmospheric modeling and many other phenomena. Since the seminal work [1], a large body of literature has been devoted to the tensor-based quadrilateral/hexahedral element methods (QSEM) (see, e.g., [2]). Recently, some progress has also been made in the triangular/tetrahedral spectral/hp element methods (TSEM), and the current approaches are mainly based on (1) the Koornwinder-Dubiner polynomials [3,4]; (2) non-polynomial on triangular

\author{
L.-L. Wang $(\varangle)$ \\ Division of Mathematical Sciences, School of Physical and Mathematical Sciences, \\ Nanyang Technological University, Singapore 637371 \\ e-mail: lilian@ntu.edu.sg \\ Y. Li \\ College of Mathematics and Computing Science, Changsha University of Science and Technology, \\ Hunan 410004, China \\ e-mail: liyouyun8@hotmail.com \\ H. Li \\ Institute of Software, Chinese Academy of Sciences, Beijing 100190, China \\ e-mail: hynli@mail.rdcps.ac.cn \\ H. Ma \\ Department of Mathematics, College of Sciences, Shanghai University, Shanghai 200444, China \\ e-mail: hpma@shu.edu.cn
}



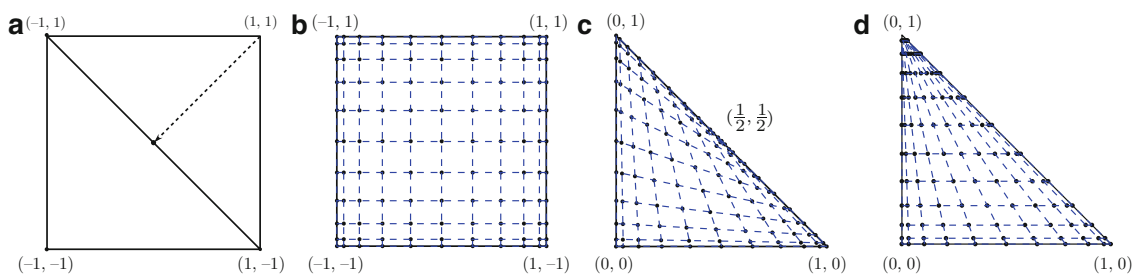

Fig. 1 (a) Illustration of the mapping (1) from the square $\mathbf{Q}$ onto the triangle $\{(x, y):-1 \leq$ $x, y ; x+y \leq 0\}$; (b) tensorial Legendre-Gauss-Lobatto (LGL) grids on $\mathbf{Q}$; (c) mapped LGL grids on $\mathbf{T}$ using the mapping (3); (d) mapped LGL grids on T using Duffy's transform in [4]

elements [5,6]; or (3) special nodal points $[7,8]$. In the first approach, the collapsed mapping (i.e., the Duffy's transform) is used to generate warped tensorial orthogonal polynomials on triangles/tetrahedra from the tensorial polynomial bases on rectangles/hexahedra. The second technique is also based on such a mapping to generate rational basis functions rather than polynomials. It is known that the Duffy's transform collapses one edge/face of the reference rectangle/hexahedron into a vertex of the triangle/tetrahedron, so the computational grids are severely clustered near the singular vertex.

This note aims to introduce a new rectangle-to-triangle mapping, which pulls one edge (at the middle point) of the triangle to two edges of the reference rectangle (cf. Fig. 1a). In contrast with the collapsed mapping, such a mapping is one-to-one, and leads to a more reasonable distribution on the triangle (cf. Fig. 1c, d). Most importantly, with a slight modification of the nodal Lagrange polynomial basis on the reference rectangle, we can derive a nodal basis (formed by irrational functions) on the triangles, which allows for an efficient implementation as with the QSEM. In a nutshell, we can view a triangular element as a deformed rectangular element, and demonstrate that the numerical issues induced by the deformation can be handled effectively. Significantly, this provides a great flexibility for the mesh generation and improves the performance of QSEM. Typically, allowing the elements being triangles along the boundaries, one can handle more complex computational domains with more regular meshes.

In this note, we first introduce the mapping and the nodal basis, then consider the implementation on a triangle, followed by the extensions to three dimensions and spectral-element methods.

\section{Rectangle-to-Triangle Mapping and Nodal Basis}

Hereafter, $(x, y)$ is the Cartesian coordinate of a generic point in a triangle, while $(\xi, \eta)$ represents the Cartesian coordinate of a point in the reference square: $\mathbf{Q}=$ $(-1,1)^{2}$. Given the vertex coordinates $\left\{\left(x_{A}, y_{A}\right),\left(x_{B}, y_{B}\right),\left(x_{D}, y_{D}\right)\right\}$ of a triangle $\triangle_{A B D}$, we can one-to-one map the square $\mathbf{Q}$ to the triangle region through

$$
(x, y)=\left(x_{A}, y_{A}\right) \frac{(1-\xi)(1-\eta)}{4}+\left(x_{B}, y_{B}\right) \frac{(1+\xi)(3-\eta)}{8}+\left(x_{D}, y_{D}\right) \frac{(3-\xi)(1+\eta)}{8} .
$$


Under this mapping, the vertices $(-1,-1),(1,-1)$ and $(-1,1)$ of $\mathbf{Q}$ correspond to the vertices $A, B, D$ of $\triangle_{A B D}$, respectively, while the middle point $C$ of the edge $B D$ is the image of the vertex $(1,1)$ of $\mathbf{Q}$. Hence, this mapping deforms two edges $(\xi=1$ and $\eta=1)$ of $\mathbf{Q}$ into one single edge $(B D)$ of $\triangle_{A B D}$. An illustration of such a one-to-one correspondence is depicted in Fig. 1.

To be more specific, we confine ourselves to the special triangle:

$$
\mathbf{T}:=\{(x, y): 0<x, y<1,0<x+y<1\},
$$

and in this case, the mapping (1) (with $B D$ being the hypotenuse) takes the form:

$$
x=\frac{1}{8}(1+\xi)(3-\eta), \quad y=\frac{1}{8}(3-\xi)(1+\eta), \quad \forall(\xi, \eta) \in \mathbf{Q},
$$

with the inversion

$$
\left\{\begin{array}{l}
\xi=1+x-y-\sqrt{(x-y)^{2}+4(1-x-y)}, \\
\eta=1-x+y-\sqrt{(x-y)^{2}+4(1-x-y)},
\end{array} \quad \forall(x, y) \in \mathbf{T} .\right.
$$

Under this mapping, we have

$$
\frac{\partial x}{\partial \xi}=\frac{3-\eta}{8}, \quad \frac{\partial x}{\partial \eta}=-\frac{1+\xi}{8}, \quad \frac{\partial y}{\partial \xi}=-\frac{1+\eta}{8}, \quad \frac{\partial y}{\partial \eta}=\frac{3-\xi}{8},
$$

so the Jacobian determinant is given by

$$
J=\operatorname{det}\left(\frac{\partial(x, y)}{\partial(\xi, \eta)}\right)=\frac{2-\xi-\eta}{16} .
$$

In the sequel, we always associate a function $u$ in $\mathbf{T}$ with a unction $\tilde{u}$ in $\mathbf{Q}$ via the mapping (3): $\tilde{u}(\xi, \eta)=u(x, y)$ and likewise for $\tilde{v}$ etc. One verifies that

$$
\nabla u=\left(\partial_{x} u, \partial_{y} u\right)=\frac{2}{2-\xi-\eta}\left((3-\xi) \partial_{\xi} \tilde{u}+(1+\eta) \partial_{\eta} \tilde{u},(1+\xi) \partial_{\xi} \tilde{u}+(3-\eta) \partial_{\eta} \tilde{u}\right):=\widetilde{\nabla} \tilde{u},
$$

and

$$
\begin{aligned}
& \iint_{\mathbf{T}} \nabla u \cdot \nabla v d x d y=\iint_{\mathbf{Q}}(\widetilde{\nabla} \tilde{u} \cdot \widetilde{\nabla} \tilde{v}) J d \xi d \eta \\
& \quad=\iint_{\mathbf{Q}}\left(G_{1}(\xi) \partial_{\xi} \tilde{u} \partial_{\xi} \tilde{v}+G_{2}(\xi, \eta)\left(\partial_{\xi} \tilde{u} \partial_{\eta} \tilde{v}+\partial_{\eta} \tilde{u} \partial_{\xi} \tilde{v}\right)+G_{1}(\eta) \partial_{\eta} \tilde{u} \partial_{\eta} \tilde{v}\right) \frac{1}{J} d \xi d \eta,
\end{aligned}
$$

where $G_{1}$ and $G_{2}$ are given by

$$
G_{1}(z)=\frac{1}{64}\left((1+z)^{2}+(3-z)^{2}\right), \quad G_{2}(\xi, \eta)=\frac{1}{8}-\frac{1}{32}(1-\xi)(1-\eta) .
$$


Consequently, the space $H^{1}(\mathbf{T})$ is mapped to the weighted space over $\mathbf{Q}$ :

$$
\widetilde{H}_{\omega}^{1}(\mathbf{Q}):=\left\{\tilde{u} \in L_{\omega}^{2}(\mathbf{Q}): \widetilde{\nabla} \tilde{u} \in L_{\omega}^{2}(\mathbf{Q})\right\} \text { with } \omega=J,
$$

and vice verse. We observe from (7) and (8) that if $\nabla u$ is well-defined at the middle point $\left(\frac{1}{2}, \frac{1}{2}\right)$ of the hypotenuse of $\mathbf{T}$, then we have

$$
\left.\left(\frac{\partial \tilde{u}}{\partial \xi}+\frac{\partial \tilde{u}}{\partial \eta}\right)\right|_{(1,1)}=0
$$

This condition induced by the rectangle-to-triangle deformation can be viewed as an analogy of the pole condition in the polar and spherical coordinates. An essential point here is how to treat this condition effectively without loss of accuracy and implementation efficiency. For this purpose, we next construct a nodal basis for the finite-dimensional approximation space over $\mathbf{Q}$ :

$$
\widetilde{X}_{N}:=\widetilde{H}_{\omega}^{1}(\mathbf{Q}) \cap\left[\mathscr{P}_{N}\right]^{2}=\left\{\phi \in\left[\mathscr{P}_{N}\right]^{2}:\left.\left(\partial_{\xi} \phi+\partial_{\eta} \phi\right)\right|_{(1,1)}=0\right\},
$$

where $\mathscr{P}_{N}$ is the set of all algebraic polynomials of degree $\leq N$ in $(-1,1)$. Let $\left\{z_{j}\right\}_{j=0}^{N}$ (with $z_{0}=-1$ and $z_{N}=1$ ) be the Legendre-Gauss-Lobatto points, i.e., the zeros of the polynomial $\left(1-z^{2}\right) L_{N}^{\prime}(z)$, where $L_{N}$ is the Legendre polynomial of degree $N$. Let $\left\{h_{j}\right\}_{j=0}^{N}$ be the Lagrange polynomial basis associated with $\left\{z_{j}\right\}_{j=0}^{N}$, and denote $d_{j k}=h_{k}^{\prime}\left(z_{j}\right)$. Define

$$
\tilde{h}_{j}(z):=h_{j}(z)-\frac{d_{N j}}{2 d_{N N}} h_{N}(z), \quad 0 \leq j \leq N-1 .
$$

It is clear that $h_{j}(z) \in \mathscr{P}_{N}$ and

$$
\tilde{h}_{j}\left(z_{k}\right)=\delta_{k j}, \quad \tilde{h}_{j}(1)=-\frac{d_{N j}}{2 d_{N N}}, \quad \tilde{h}_{j}^{\prime}(1)=\frac{d_{N j}}{2}, \quad 0 \leq k, j \leq N-1 .
$$

Setting

$$
\psi_{i j}(\xi, \eta)= \begin{cases}h_{i}(\xi) h_{j}(\eta), & 0 \leq i, j \leq N-1 \\ \tilde{h}_{i}(\xi) h_{N}(\eta), & 0 \leq i \leq N-1 ; j=N \quad(\text { edge }: \xi=1) \\ h_{N}(\xi) \tilde{h}_{j}(\eta), & i=N, \quad 0 \leq j \leq N-1 \quad(\text { edge }: \eta=1)\end{cases}
$$

and

$$
\Upsilon_{N}:=\{(i, j): 0 \leq i, j \leq N \text { but }(i, j) \neq(N, N)\},
$$

we find from (14) that all the $\psi_{i j}$ satisfy (11) and 


$$
\widetilde{X}_{N}=\operatorname{span}\left\{\psi_{i j}:(i, j) \in \Upsilon_{N}\right\} \Rightarrow \operatorname{dim}\left(\widetilde{X}_{N}\right)=(N+1)^{2}-1 .
$$

It is seen that we modified the usual tensorial nodal basis $\left\{h_{i}(\xi) h_{j}(\eta)\right\}_{i, j=0}^{N}$ along the edges: $\xi=1$ and $\eta=1$ of $\mathbf{Q}$ so as to meet the condition (11) at the singular point. In view of (14), $\left\{\psi_{i j}\right\}_{i, j \in \Upsilon_{N}}$ forms a nodal basis of $\widetilde{X}_{N}$. More precisely, we have

$$
\psi_{i j}\left(\xi_{p}, \eta_{q}\right)=\delta_{p i} \delta_{q j}, \quad \forall(i, j),(p, q) \in \Upsilon_{N},
$$

where $\left\{\xi_{k}=\eta_{k}=z_{k}\right\}_{k=0}^{N}$ are the LGL points as before.

The above nodal basis is complete in $\widetilde{H}_{\omega}^{1}(\mathbf{Q})$, but in order to enforce continuity across the elements, we need to define a nodal basis function at the singular vertex $(1,1)$. Define

$$
\psi_{N N}(\xi, \eta)=\tilde{h}_{N}(\xi) \tilde{h}_{N}(\eta)
$$

where $\tilde{h}_{N}(z)=\left(1+d_{N N}-z d_{N N}\right) h_{N}(z)$. Observe that $\psi_{N N} \notin \widetilde{X}_{N}$ and satisfies

$$
\left.\left(\frac{\partial \psi_{N N}}{\partial \xi}+\frac{\partial \psi_{N N}}{\partial \eta}\right)\right|_{(1,1)}=0, \quad \psi_{N N}\left(\xi_{p}, \eta_{q}\right)=\delta_{p N} \delta_{q N}, \quad 0 \leq p, q \leq N
$$

Hence, $\psi_{N N}$ must be linearly independent with the basis functions defined in (15). Hereafter, we update $\widetilde{X}_{N}$ by adding $\psi_{N N}$ with dimensionality $(N+1)^{2}$.

Another important property of this basis is that the singularity induced by the transform is removable in the following sense.

Lemma 1. For any $\tilde{u}, \tilde{v} \in \widetilde{X}_{N}$,

$$
\left.\{(\widetilde{\nabla} \tilde{u} \cdot \widetilde{\nabla} \tilde{v}) J\}\right|_{(1,1)}=0,
$$

where $J$ and $\widetilde{\nabla}$ are defined in (6) and (7), respectively.

Proof. For any $\tilde{u} \in \widetilde{X}_{N}$, define

$$
w(\xi, \eta):=(3-\xi) \partial_{\xi} \tilde{u}+(1+\eta) \partial_{\eta} \tilde{u},
$$

and we have

$$
\left.\left(\partial_{\xi} \tilde{u}+\partial_{\eta} \tilde{u}\right)\right|_{(1,1)}=0 \Rightarrow w(1,1)=0 .
$$

Using Taylor expansion yields

$$
w(\xi, \eta)=-(1-\xi) \partial_{\xi} w(1,1)-(1-\eta) \partial_{\eta} w(1,1)+O\left((1-\xi)^{2}+(1-\xi)(1-\eta)+(1-\eta)^{2}\right) .
$$

It is obvious that

$$
0 \leq \frac{1-\xi}{(1-\xi)+(1-\eta)} \leq 1, \quad 0 \leq \frac{(1-\xi)^{2}}{(1-\xi)+(1-\eta)} \leq 1-\xi, \quad \forall(\xi, \eta) \in \mathbf{Q}
$$


and likewise for $1-\eta$ and other terms in big " $O$," so we have

$$
\left.\frac{w(\xi, \eta)}{2-\xi-\eta}\right|_{(1,1)}=\text { constant }
$$

Consequently, $\widetilde{\nabla} \tilde{u}$ is well-defined at $(1,1)$, so is $\widetilde{\nabla} \tilde{v}$. As the determinant Jacobian $J$ vanishes at $(1,1),(20)$ holds.

\section{Implementations and Numerical Results}

To test the approximation property of the foregoing nodal basis, we now implement the spectral methods for the elliptic equation in $\mathbf{T}$ :

$$
-\operatorname{div}(a \operatorname{grad} u)+b u=f \quad \text { in } \mathbf{T} ; \quad u=0 \quad \text { on } \Gamma_{1} ; \quad \frac{\partial u}{\partial \mathrm{n}}=g \quad \text { on } \Gamma_{2},
$$

where $a, b$ and $f$ are given functions satisfying

$$
a \in L^{\infty}(\mathbf{T}), \quad a(x, y) \geq a_{0}>0, \quad b(x, y) \geq 0, \quad \forall(x, y) \in \overline{\mathbf{T}},
$$

for certain constant $a_{0}, \Gamma_{1}$ (resp. $\Gamma_{2}$ ) consists of the edges $x=0$ and $y=0$ (resp. $x+y=1)$, and $\mathrm{n}$ is the unit outer normal vector along $\Gamma_{2}$. The weak formulation of (21) is to find $u \in H_{\Gamma_{1}}^{1}(\mathbf{T}):=\left\{u \in H^{1}(\mathbf{T}):\left.u\right|_{\Gamma_{1}}=0\right\}$ such that

$$
\mathscr{B}(u, v)=(a \nabla u, \nabla v)_{\mathbf{T}}+(b u, v)_{\mathbf{T}}=(f, v)_{\mathbf{T}}+(a g, v)_{\Gamma_{2}}, \quad \forall v \in H_{\Gamma_{1}}^{1}(\mathbf{T}),
$$

where $(g, v)_{\Gamma_{2}}=\int_{\Gamma_{2}} g v d \gamma$.

We view $\mathbf{T}$ as a deformed triangle as a deformed quadrilateral element, and perform the numerical integration and differentiation on the reference element $\mathbf{Q}$. Define the discrete inner product associated with the usual tensorial LGL quadrature rule:

$$
\langle u, v\rangle_{N, \mathbf{T}}=\left.\sum_{0 \leq p, q \leq N}(\tilde{u} \tilde{v} J)\right|_{\left(\xi_{p}, \eta_{q}\right)} \omega_{p} \omega_{q}:=\langle\tilde{u}, \tilde{v} J\rangle_{N, \mathbf{Q}}, \quad \forall u, v \in C(\overline{\mathbf{T}}),
$$

where $\left\{\omega_{k}\right\}$ are the LGL quadrature weights associated with LGL points $\left\{\xi_{k}=\eta_{k}\right\}$. Similarly, we can define the discrete rule, denoted by $\langle\cdot, \cdot\rangle_{N, \Gamma_{2}}$, along $\Gamma_{2}$, which sums the contributions from two edges $\xi=1$ and $\eta=1$.

The Galerkin approximation with numerical integration (GaNI) of (23) is to find $u_{N} \in V_{N}:=\operatorname{span}\left\{\phi_{i j}(x, y)=\psi_{i j}(\xi, \eta) \in \widetilde{X}_{N}: 1 \leq i, j \leq N\right\}$ such that

$$
\begin{aligned}
\mathscr{B}_{N}\left(u_{N}, v_{N}\right) & =\left\langle a \nabla u_{N}, \nabla v_{N}\right\rangle_{N, \mathbf{T}}+\left\langle b u_{N}, v_{N}\right\rangle_{N, \mathbf{T}} \\
& =\left\langle f, v_{N}\right\rangle_{N, \mathbf{T}}+\left\langle a g, v_{N}\right\rangle_{N, \Gamma_{2}}, \quad \forall v_{N} \in V_{N} .
\end{aligned}
$$


Table $1 L^{2}$-error, Max -error and the error at the middle point $(1 / 2,1 / 2)$ for Example 1

\begin{tabular}{llllllll}
\hline & \multicolumn{3}{c}{ Without (18) } & & \multicolumn{3}{c}{ With $(18)$} \\
\cline { 2 - 3 } \cline { 6 - 8 }$N$ & \multicolumn{1}{c}{$L^{2}$} & Max & $(1 / 2,1 / 2)$ & & \multicolumn{1}{c}{$L^{2}$} & \multicolumn{1}{c}{ Max } & $(1 / 2,1 / 2)$ \\
\hline 4 & $2.186 \mathrm{e}-3$ & $5.624 \mathrm{e}-3$ & $2.782 \mathrm{e}-4$ & & $2.186 \mathrm{e}-3$ & $5.624 \mathrm{e}-3$ & $3.538 \mathrm{e}-3$ \\
8 & $4.784 \mathrm{e}-7$ & $3.693 \mathrm{e}-6$ & $1.733 \mathrm{e}-6$ & & $4.784 \mathrm{e}-7$ & $4.781 \mathrm{e}-6$ & $4.781 \mathrm{e}-6$ \\
12 & $1.180 \mathrm{e}-10$ & $1.486 \mathrm{e}-9$ & $1.614 \mathrm{e}-10$ & & $1.180 \mathrm{e}-10$ & $1.486 \mathrm{e}-9$ & $2.070 \mathrm{e}-10$ \\
16 & $3.422 \mathrm{e}-14$ & $3.457 \mathrm{e}-13$ & $6.006 \mathrm{e}-14$ & & $3.422 \mathrm{e}-14$ & $3.457 \mathrm{e}-13$ & $1.267 \mathrm{e}-13$ \\
20 & $2.075 \mathrm{e}-14$ & $9.892 \mathrm{e}-14$ & $2.231 \mathrm{e}-14$ & & $2.075 \mathrm{e}-14$ & $9.892 \mathrm{e}-14$ & $2.120 \mathrm{e}-14$ \\
24 & $1.344 \mathrm{e}-13$ & $6.971 \mathrm{e}-13$ & $3.363 \mathrm{e}-14$ & & $1.344 \mathrm{e}-13$ & $6.971 \mathrm{e}-13$ & $3.386 \mathrm{e}-14$ \\
28 & $2.109 \mathrm{e}-13$ & $1.000 \mathrm{e}-12$ & $1.841 \mathrm{e}-13$ & & $2.109 \mathrm{e}-13$ & $1.000 \mathrm{e}-12$ & $1.874 \mathrm{e}-13$ \\
32 & $8.701 \mathrm{e}-14$ & $3.211 \mathrm{e}-13$ & $1.387 \mathrm{e}-13$ & $8.701 \mathrm{e}-14$ & $3.211 \mathrm{e}-13$ & $1.371 \mathrm{e}-13$ \\
\hline
\end{tabular}

Some remarks are in order. Firstly, we could remove the extra basis function (18) at the singular point from $V_{N}$ for a single triangle. Moreover, in view of Lemma 1, the physical values of the terms at the singular point vanish. The well-posedness of (23) and (25) can be proved by a standard argument. basis.

We next present some examples to illustrate the approximability of the nodal

Example 1. We consider (21) with $a(x, y)=x+2, b(x, y)=x+y$ and a smooth exact solution:

$$
u(x, y)=e^{x+y-1} \sin \left(3 y\left(y-\frac{\sqrt{3}}{2} x+\frac{\sqrt{3}}{4}\right)\right) .
$$

We tabulate in Table 1 the maximum pointwise and discrete $L^{2}$ errors on $\mathbf{T}$ for various $N$. Particularly, we single out the errors at the singular middle point $(1 / 2,1 / 2)$, and list the numerical errors for the scheme (25) with or without the extra basis function (18). We observe an exponential decay of the errors with a convergence behavior similar to that of the quadrilateral element case using tensorial Lagrange polynomial basis (see, e.g., Fig. 2.17 in [9]). Moreover, the presence of the basis function (18) essentially does not affect the performance of the scheme (25).

Example 2. We consider (21) with $a=b=1$ and test the exact solution with a finite regularity

$$
u(x, y)=(1-x-y)^{\frac{5}{2}}\left(e^{x y}-1\right) \in H^{3-\epsilon}(\mathbf{T}), \quad \epsilon>0 .
$$

We list in Table 2 the errors for various $N$, which indicates an algebraic decay of the errors with a convergence rate around $O\left(N^{-3}\right)$. It is known that for a tensorbased spectral approximation on a rectangle, the theoretical order of convergence is $O\left(N^{-3+\varepsilon}\right)$. Although we have not provided the analysis, the proposed scheme really enjoys a similar convergence behavior. 
Table $2 L^{2}$-error, Max -error and the error at the middle point $(1 / 2,1 / 2)$ for Example 2

\begin{tabular}{lccccccc}
\hline \multirow{2}{*}{$N$} & \multicolumn{3}{c}{ Without $(18)$} & & \multicolumn{3}{c}{ With $(18)$} \\
\cline { 2 - 4 } \cline { 6 - 7 } \cline { 6 - 8 } & $L^{2}$ & Max & $(1 / 2,1 / 2)$ & & $L^{2}$ & Max & $(1 / 2,1 / 2)$ \\
\hline 15 & $2.866 \mathrm{e}-6$ & $1.018 \mathrm{e}-5$ & $5.895 \mathrm{e}-6$ & & $2.866 \mathrm{e}-6$ & $1.018 \mathrm{e}-5$ & $5.895 \mathrm{e}-6$ \\
30 & $3.410 \mathrm{e}-7$ & $1.203 \mathrm{e}-6$ & $7.045 \mathrm{e}-7$ & & $3.410 \mathrm{e}-7$ & $1.203 \mathrm{e}-6$ & $7.045 \mathrm{e}-7$ \\
45 & $9.940 \mathrm{e}-8$ & $3.513 \mathrm{e}-7$ & $2.054 \mathrm{e}-7$ & & $9.940 \mathrm{e}-8$ & $3.513 \mathrm{e}-7$ & $2.054 \mathrm{e}-7$ \\
60 & $4.158 \mathrm{e}-8$ & $1.469 \mathrm{e}-7$ & $8.600 \mathrm{e}-8$ & & $4.159 \mathrm{e}-8$ & $1.468 \mathrm{e}-7$ & $8.598 \mathrm{e}-8$ \\
75 & $2.101 \mathrm{e}-8$ & $7.599 \mathrm{e}-8$ & $4.757 \mathrm{e}-8$ & & $2.118 \mathrm{e}-8$ & $7.486 \mathrm{e}-8$ & $4.375 \mathrm{e}-8$ \\
90 & $1.221 \mathrm{e}-8$ & $4.318 \mathrm{e}-8$ & $2.533 \mathrm{e}-8$ & & $1.222 \mathrm{e}-8$ & $4.316 \mathrm{e}-8$ & $2.528 \mathrm{e}-8$ \\
105 & $7.669 \mathrm{e}-9$ & $2.723 \mathrm{e}-8$ & $1.620 \mathrm{e}-8$ & & $7.683 \mathrm{e}-9$ & $2.706 \mathrm{e}-8$ & $1.553 \mathrm{e}-8$ \\
120 & $1.075 \mathrm{e}-8$ & $3.472 \mathrm{e}-8$ & $2.632 \mathrm{e}-8$ & & $5.279 \mathrm{e}-9$ & $1.942 \mathrm{e}-8$ & $1.817 \mathrm{e}-8$ \\
\hline
\end{tabular}

\section{Extensions and Discussions}

A key point in the previous discussion is to one-to-one map a triangular element to the reference rectangle, and to view it as a deformed quadrilateral element. This provides some flexibility for mesh generation of QSEM. Typically, a hybrid spectral-element method can be constructed by using the triangular elements along the boundaries (with the singular edges facing the boundaries) and quadrilateral elements in the interior of the computational domains. This might lead to a more regular mesh and enhance the capability of QSEM for more complex geometries. On the other hand, the number of points on the singular edge is double of the points on the other two edges, so the singular edge should adjoin two quadrilateral elements and/or triangular elements (but share two nonsingular edges), or a triangular element (but share the singular edge). The availability of the aforementioned nodal basis makes the implementation of the hybrid spectral-element method almost as efficient as the usual QSEM.

We now discuss the extensions to tetrahedral elements. Let $\mathrm{T}$ be a tetrahedron with vertices $A, B, C$ and $D$. Denote by $\mathrm{Q}$ the reference cube $\{(\xi, \eta, \zeta):-1<\xi, \eta$, $\zeta<1\}$. The counterpart of (1) reads

$$
\begin{aligned}
(x, y, z)= & \left(x_{A}, y_{A}, z_{A}\right) \frac{(1-\xi)(1-\eta)(1-\zeta)}{8}+\left(x_{B}, y_{B}, z_{B}\right) \frac{(1+\xi)(7-2 \eta-2 \zeta+\eta \zeta)}{24} \\
& +\left(x_{C}, y_{C}, z_{C}\right) \frac{(1+\eta)(7-2 \xi-2 \zeta+\xi \zeta)}{24}+\left(x_{D}, y_{D}, z_{D}\right) \frac{(1+\zeta)(7-2 \xi-2 \eta+\xi \eta)}{24}
\end{aligned}
$$

which is one-to-one and maps the vertices $(-1,-1,-1),(1,-1,-1),(-1,1,-1)$ and $(-1,-1,1)$ of $\mathrm{Q}$ to the vertices of $A, B, C$ and $D$ of the tetrahedron T, respectively, while the images of vertices $(-1,1,1),(1,-1,1),(1,1,-1)$ and $(1,1,1)$ of $\mathrm{Q}$ are the middle points of the sides $C D, D B, B C$ and the barycenter of the face $\triangle B C D$, respectively. An illustration of this mapping is depicted in Fig. 2a.

In particular, for the specific tetrahedron

$$
\mathrm{T}=\{(x, y, z): 0 \leq x, y, z ; x+y+z \leq 1\},
$$


a

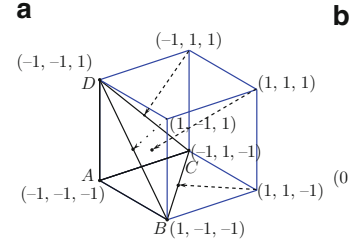

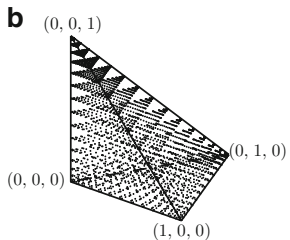

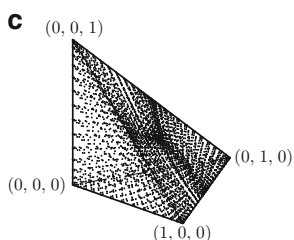

d

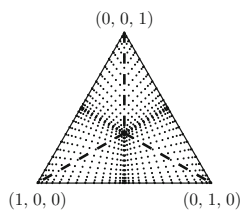

Fig. 2 (a) Illustration of the mapping from the cube $Q=(-1,1)^{3}$ and the tetrahedron $\{(x, y, z)$ : $-1<x, y, z ; x+y+z<-1\}$; (b) mapped tensorial Legendre-Gauss-Lobatto (LGL) grids on T based on the Duffy's mapping in [4]; (c) mapped LGL grids on T using the mapping (28); (d) distribution of the grids on the singular face $x+y+z=1$ of T

the mapping takes the form

$$
\left\{\begin{array}{l}
x=\frac{1}{24}(1+\xi)(7-2 \eta-2 \zeta+\eta \zeta) \\
y=\frac{1}{24}(1+\eta)(7-2 \xi-2 \zeta+\xi \zeta) \\
z=\frac{1}{24}(1+\zeta)(7-2 \xi-2 \eta+\xi \eta)
\end{array}\right.
$$

We plot in Fig. 2b, c the distributions of the mapped tensorial Legendre-GaussLobatto grids on T based on the Duffy's mapping and the mapping (28). The Duffy's mapping collapses one face of $Q$ into a vertex of $T$, so many collocation points cluster near the singular vertex, which turn out to be wasted. In contrast, the use of (28) leads to a more reasonable grid distribution. Like (11), similar conditions induced by the mapping should be imposed along the three lines that connect the barycenter and the middle points of three side of the singular face of T. Hence, the construction of the nodal basis is much more involved.

We shall report the numerical analysis and the applications of such spectralelement methods in a forthcoming paper.

Acknowledgements The work of the author Youyun Li was supported by Scientific Research Foundation for Returned Scholars, Ministry of Education of China. The work of the author LiLian Wang was supported by Singapore AcRF Tier 1 Grant RG58/08, Singapore MOE Grant T207B2202, Singapore NRF2007IDM-IDM002-010, and Leading Academic Discipline Project of Shanghai Municipal Education Commission Grant J50101. The work of the author Huiyuan Li was supported by National Natural Science Foundation of China (NSFC) Grants 10601056 and 10971212. The work of the author Heping Ma was supported by National Natural Science Foundation of China (NSFC) Grant 60874039.

\section{References}

1. Rønquist, M., Patera T.: A Legendre spectral element method for the incompressible NavierStokes equations. Brunswick, pp. 318-326. Friedrich Vieweg und Sohn, Germany (1988)

2. Deville, M.O., Fischer, P.F, Mund, E.H.: High-order methods for incompressible fluid flow. Cambridge University Press, London (2002) 
3. Dubiner, M.: Spectral methods on triangles and other domains. J. Sci. Comput., 6, 345-390 (1991)

4. Karniadakis, G.E., Sherwin, S.J.: Spectral $/ h p$ element methods for computational fluid dynamics. Oxford University Press, New York (2005)

5. Heinrichs, W., Loch, B.I., Spectral schemes on triangular elements. J. Comput. Phys., 173, 279-301 (2001)

6. Shen J., Wang, L.L., Li H.Y.: A triangular spectral element method using fully tensorial rational basis functions. SIAM. J. Numer. Anal., 47, 1619-1650 (2009)

7. Hesthaven, J.S.: From electrostatics to almost optimal nodal sets for polynomial interpolation in a simplex. SIAM J. Numer. Anal., 35, 655-676 (1998)

8. Taylor, M.A., Wingate, B.A., Vincent, R.E.: An algorithm for computing Fekete points in the triangle. SIAM J. Numer. Anal., 38, 1707-1720 (2000)

9. Canuto, C., Hussaini, M.Y., Quarteroni, A., and Zang, T.A.: Spectral methods: Fundamentals in single domains. Scientific Computation. Springer, Berlin, 2006 